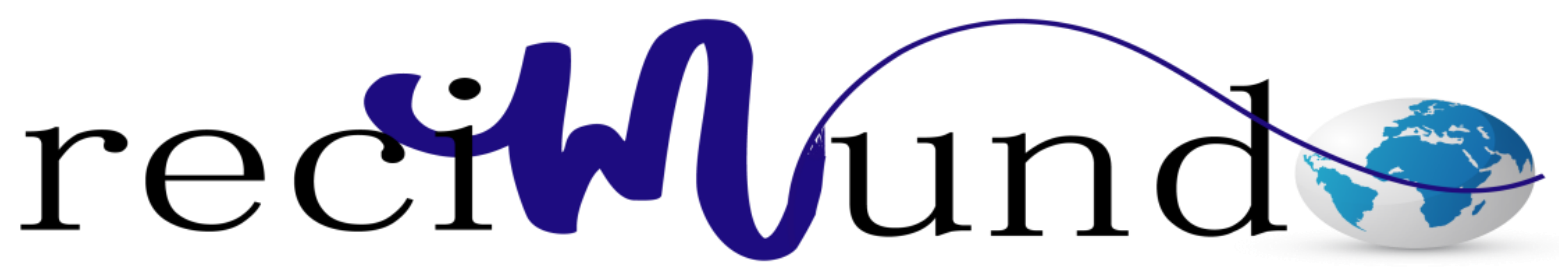

Revista Científica Mundo de la Investigación y el Conocimiento

Milka María Lozano Domínguez a; Germania Marivel Vargas Aguilar ${ }^{\text {b; }}$ Crelia Mariana Tufiño Gavidia ${ }^{c}$; María Eiralda Mieles Moreira ${ }^{\mathrm{d}}$

Cuidados de enfermería en la atención de neonatos con síndrome de abstinencia

Nursing care in the care of neonates with withdrawal sindrome

Revista Científica Mundo de la Investigación y el Conocimiento. Vol. 2 núm.3, julio, ISSN: 2588-073X, 2018, pp. 611-627

DOI: 10.26820/recimundo/2.(3).julio.2018.648-627

Editorial Saberes del Conocimiento

Recibido: 05/04/2018

Aceptado: 15/02/2018

Publicado: 30/07/2018

Correspondencia: milka.lozanod@ug.edu.ec

a. Docente -Investigador Universidad de Guayaquil; milka.lozanod@ug.edu.ec

b. Docente -Investigador Universidad de Guayaquil; germania.vargasa@ug.edu.ec

c. Docente -Investigador Universidad de Guayaquil; mariana.tufinog@ug.edu.ec

d. Universidad de Guayaquil; Líder de Servicio de Neonatología Hospital Materno Infantil del Guasmo; mamimo67@hotmail.com 


\section{Cuidados de enfermería en la atención de neonatos con síndrome de abstinencia}

Vol. 2, núm. 3., (2018)

Milka María Lozano Domínguez; Germania Marivel Vargas Aguilar; Crelia Mariana Tufiño Gavidia; María Eiralda Mieles Moreira

\section{RESUMEN}

La planificación de los cuidados del o la recién nacida hija de una madre afectada por las drogas supone un desafío para el equipo de salud, dado que la individualización de los cuidados y la activación de las redes de apoyo son aspectos esenciales. Una vez que se da el ingreso de la madre al Servicio de Obstetricia se debe valorar la existencia de síndrome de abstinencia: si esta es negativa, se le asigna una cama en el salón, pero si es positiva la cama asignada de preferencia debe de ser en aislamiento, con el fin de brindar tratamiento para desintoxicación y los cuidados especiales que requiere. Una vez que se da el nacimiento del bebé y éste se enfrenta al ambiente externo, al dejar inconscientemente de consumir la droga, el recién nacido puede sufrir el síndrome de abstinencia a drogas (SADRN), ante tal situación Enfermería debe conocer los signos y síntomas que el neonato presenta para valorar su estado. Los signos y síntomas son muy variados, al respecto, la Asociación Española de Pediatría (AEP) destaca la presencia de hiperexcitabilidad del SNC (irritabilidad, temblores, hiperactividad, hipertonía, llanto agudo, mioclonías, convulsiones), alteraciones digestivas (regurgitaciones, vómitos, diarreas, voracidad), alteraciones vegetativas (sudoración, hipersecreción de mucosas, fiebre, erupciones cutáneas) y alteraciones cardio respiratorias (taquipnea, congestión nasal, taquicardia. Por lo que se requiere de cuidados específicos a los neonatos de un Hospital Materno Infantil de la ciudad de Guayaquil que presenta síndrome de abstinencia.

Palabras clave: Drogas, neonatos, síndrome de abstinencia, cuidados de enfermería. 


\title{
Cuidados de enfermería en la atención de neonatos con síndrome de abstinencia
}

Vol. 2, núm. 3., (2018)

Milka María Lozano Domínguez; Germania Marivel Vargas Aguilar; Crelia Mariana Tufiño Gavidia; María Eiralda Mieles Moreira

\begin{abstract}
Planning the care of the newborn or daughter of a mother affected by drugs poses a challenge to the health team, as the individualization of care and the activation of support networks are essential. Once the mother's admission to the Obstetrics Service is given, the existence of withdrawal syndrome must be assessed: if this is negative, a bed is assigned in the room, but if it is positive, the assigned bed should preferably be in isolation, in order to provide treatment for detoxification and the special care it requires. Once the baby is born and the baby is faced with the external environment, when the baby unconsciously stops using the drug, the newborn can suffer from drug withdrawal syndrome (SADRN) in the face of such a situation Nursing must know the signs and symptoms that the newborn presents to assess its status. Signs and symptoms are very varied, in this respect, the Spanish Association of Pediatrics (AEP) emphasizes the presence of CNS hyperexcitability (irritability, tremors, hyperactivity, hypertonia, acute crying, myoclonus, convulsions), digestive disorders (regurgitations, vomiting, diarrhea, voracity), vegetative alterations (sweating, mucosal hypersecretion, fever, rashes) and cardio respiratory alterations (tachypnea, nasal congestion, tachycardia.) Therefore, a care guide for neonates of the Specialized Hospital which has withdrawal syndrome, both for medical assisting professionals and for parents.
\end{abstract}

Keywords: Drugs, neonates, withdrawal syndrome, nursing care. 


\section{Cuidados de enfermería en la atención de neonatos con síndrome de abstinencia}

Vol. 2, núm. 3., (2018)

Milka María Lozano Domínguez; Germania Marivel Vargas Aguilar; Crelia Mariana Tufiño Gavidia; María Eiralda Mieles Moreira

\section{Introducción.}

El preocupante aumento de la drogadicción en la sociedad actual no excluye a la mujer embarazada. El consumo y dependencia de sustancias adictivas se ha convertido en un serio problema de salud pública por la alta morbilidad maternofetal. El consumo de sustancias tóxicas, tanto legales como ilegales, provoca una situación de riesgo para la salud de la madre, el feto y el recién nacido. Además, generalmente la gestante consumidora de drogas, no utiliza una única sustancia adictiva, sino que con frecuencia asocia varias, consume tabaco y/o alcohol, está mal nutrida, padece algún tipo de infección (VHB, VHC, VIH...) y suele llevar un mal control prenatal. Por todo ello, el embarazo pasa a ser una situación de alto riesgo.

El consumo de drogas legales o ilegales en la población general y, en particular, en las mujeres en edad fértil o durante el embarazo es una circunstancia que ocasiona alteraciones psicosociales y repercusiones clínicas para los recién nacidos $(1,2,3)$. Durante la gestación, estas sustancias pasan desde el torrente sanguíneo de la madre al feto a través de la placenta, produciendo también adicción en este (4). Se estima que uno de cada diez recién nacidos vivos puede haber estado expuesto a drogas durante el período intrauterino $(5,6)$.

En el momento del nacimiento, la droga deja de estar disponible, produciendo una hiperestimulación del sistema nervioso del recién nacido, dando lugar en más del $66 \%$ de los casos al denominado Síndrome de Abstinencia Neonatal (SAN) (7). El SAN se define como un conjunto de síntomas que experimenta el neonato tras la retirada de drogas que causan adicción y que pueden evidenciarse en mayor o menor gravedad a nivel del sistema nervioso (hipertonía, temblores, 


\section{Cuidados de enfermería en la atención de neonatos con síndrome de abstinencia}

Vol. 2, núm. 3., (2018)

Milka María Lozano Domínguez; Germania Marivel Vargas Aguilar; Crelia Mariana Tufiño Gavidia; María Eiralda Mieles Moreira

irritabilidad, febrícula, etc...), gastrointestinal (diarrea, vómitos, deglución dismadura...), y otras manifestaciones (taquipnea, excoriación de la piel e irregularidades de la conducta) $(5,7,8)$.

Según la organización mundial de la salud (OMS) El síndrome de abstinencia neonatal (SAN) debido al retiro de opiáceos puede dar lugar a trastornos en la relación madre-hijo, anomalías durante el sueño o la vigilia, dificultades en la alimentación, pérdida de peso y convulsiones. Los tratamientos utilizados para paliar los síntomas y reducir la morbilidad incluyen los opiáceos, los sedantes y los tratamientos no farmacológicos.

Entre el 2005 y 2010 en EEUU, más del 14\% de las mujeres embarazadas consumieron opioides durante el embarazo y en el 2011 más del 1\% de las embarazadas utilizaron un opiáceo o heroína ilícitamente. En el 2012, la incidencia del SAN en los EEUU alcanzo el 5.8 por 1000 nacimientos. (9) En Latinoamérica, el monoconsumo de drogas ilegales es del $66.5 \%$ de la población entre 16 y 64 años, con predominio del cannabis. (10)

Ecuador no está al margen de la incidencia del SAN, es así que el diario electrónico infosanitaria relata "A lo largo de este año, se ha atendido 16 casos de síndrome de abstinencia en bebés en la Maternidad pública. Las autoridades afirman que el 70\% de los recién nacidos son hijos de madres adolescentes que consumen varias sustancias, entre ellas la denominada " $\mathrm{H}$ ", y que siete de cada diez niños que nacieron con síndrome de abstinencia en esta maternidad son de madres adolescentes. En 2015, nacieron 22 bebés con el síndrome de abstinencia de las drogas en la zona 8, que comprende los cantones de Guayaquil, Durán y Samborondón, y hasta abril de este año, 16 recién nacidos registraron este padecimiento". (11) 


\section{Cuidados de enfermería en la atención de neonatos con síndrome de abstinencia}

Vol. 2, núm. 3., (2018)

Milka María Lozano Domínguez; Germania Marivel Vargas Aguilar; Crelia Mariana Tufiño Gavidia; María Eiralda Mieles Moreira

El conocimiento de la clínica, la valoración de las manifestaciones y los cuidados de enfermería que se prestan a estos neonatos contribuyen de manera importante al bienestar del recién nacido y por tanto a su recuperación (2). Conocer la situación actual de este problema nos aportaría elementos útiles para visualizar su existencia y el abordaje integral. Por lo que nos planteamos como objetivo de esta investigación determinar el proceso de atención de enfermería en el cuidado de neonatos de madres consumidoras de heroína en el hospital materno infantil Mariana de Jesús como una guía de cuidado para los profesionales médicos y para los familiares.

\section{Métodos.}

Se utilizó una investigación cualitativa de tipo descriptiva analítica con componentes retrospectivos porque solo se basa en la descripción de los sucesos y los análisis de las situaciones. Se utilizó la encuesta y la técnica de observación directa para la captación de información para la ejecución del proyecto y el informe final además de la obtención de datos de registro a través de la historia clínica.

Se realizó la encuesta a 40 personas que incluyen enfermeras y pacientes del Hospital Especializado Mariana de Jesús. Las encuestas aplicadas a estos dos grupos tienen diferente finalidad de respuesta; a los profesionales de enfermería se les aplico una encuesta utilizando como instrumento un cuestionario con 11 preguntas cerradas y el cuestionario aplicado a las madres consumidoras de heroína con 8 preguntas cerradas.

Previo a la realización del estudio se solicitó la firma del consentimiento informado y se entregó un oficio con el tema del proyecto al director del Hospital Materno Infantil de Guayaquil por lo cual se determinó los parámetros de la investigación asegurando la confidencialidad. 


\section{Cuidados de enfermería en la atención de neonatos con síndrome de abstinencia}

Vol. 2, núm. 3., (2018)

Milka María Lozano Domínguez; Germania Marivel Vargas Aguilar; Crelia Mariana Tufiño Gavidia; María Eiralda Mieles Moreira

\section{Resultados.}

\section{Gráfico $N^{\circ} 1$ - diagnóstico de enfermería según estado de salud del neonato}

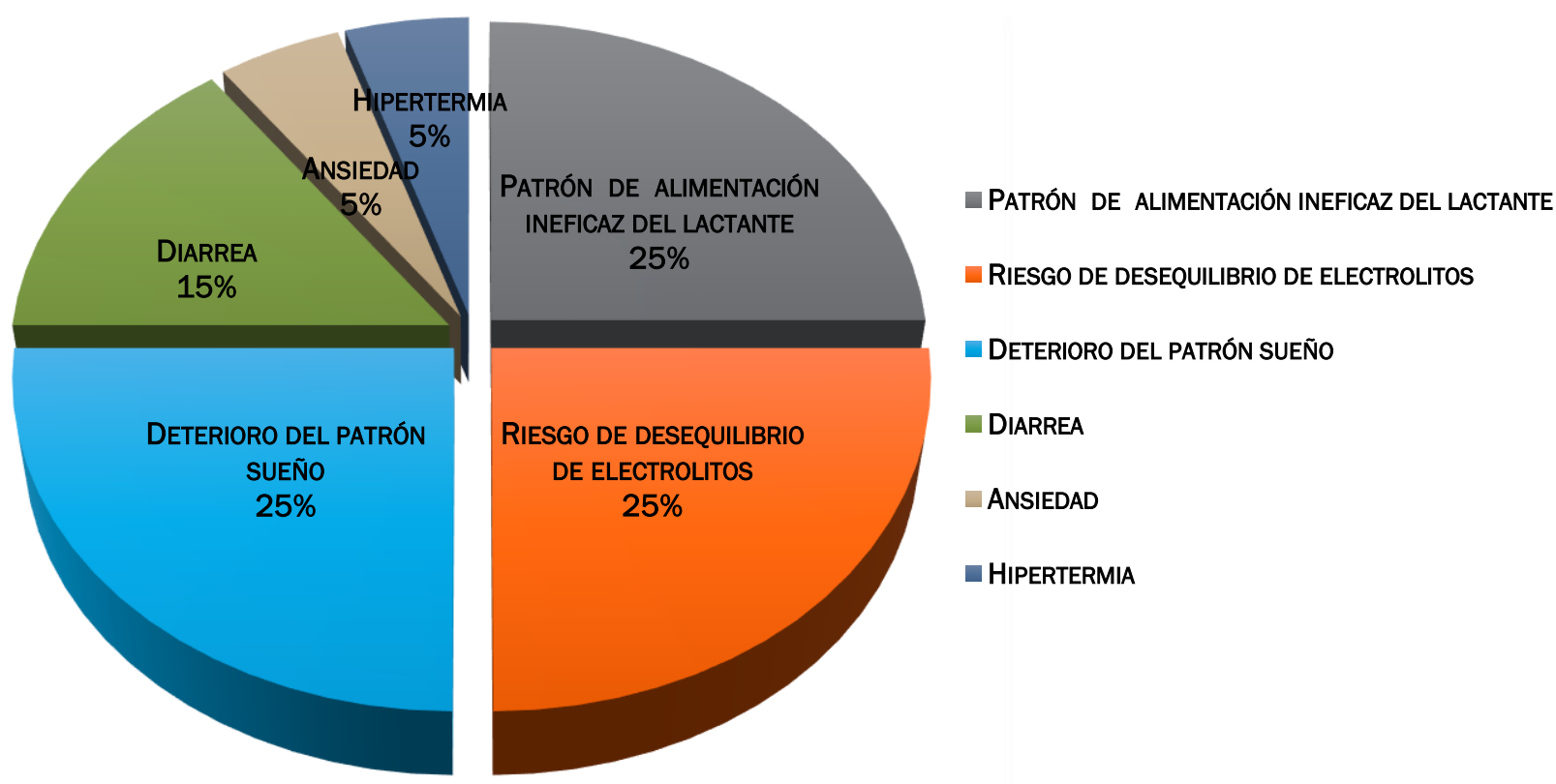

Fuente: Hospital Materno Infantil - Área de neonatología

Elaborado por: Autores

Análisis: El siguiente gráfico representa los diagnósticos de enfermería según el estado de salud del neonato. Los datos obtenidos demuestran que las etiquetas diagnósticas de enfermería más utilizadas son riesgo de desequilibrio de electrolitos, deterioro del patrón del sueño y patrón de alimentación ineficaz con 5 neonatos para cada etiqueta que representa el 25\% cada una; 3 neonatos con etiqueta diagnóstica de diarrea que representa el 15\% y 1 neonato con etiqueta de hipertermia y 1 con etiqueta de ansiedad que representa el 5\% cada una. 


\section{Cuidados de enfermería en la atención de neonatos con síndrome de abstinencia}

Vol. 2, núm. 3., (2018)

Milka María Lozano Domínguez; Germania Marivel Vargas Aguilar; Crelia Mariana Tufiño Gavidia; María Eiralda Mieles Moreira

\section{Gráfico $N^{\circ} 2$ - condiciones de salud del neonato con síndrome de abstinencia (según escala de}

finnegan)

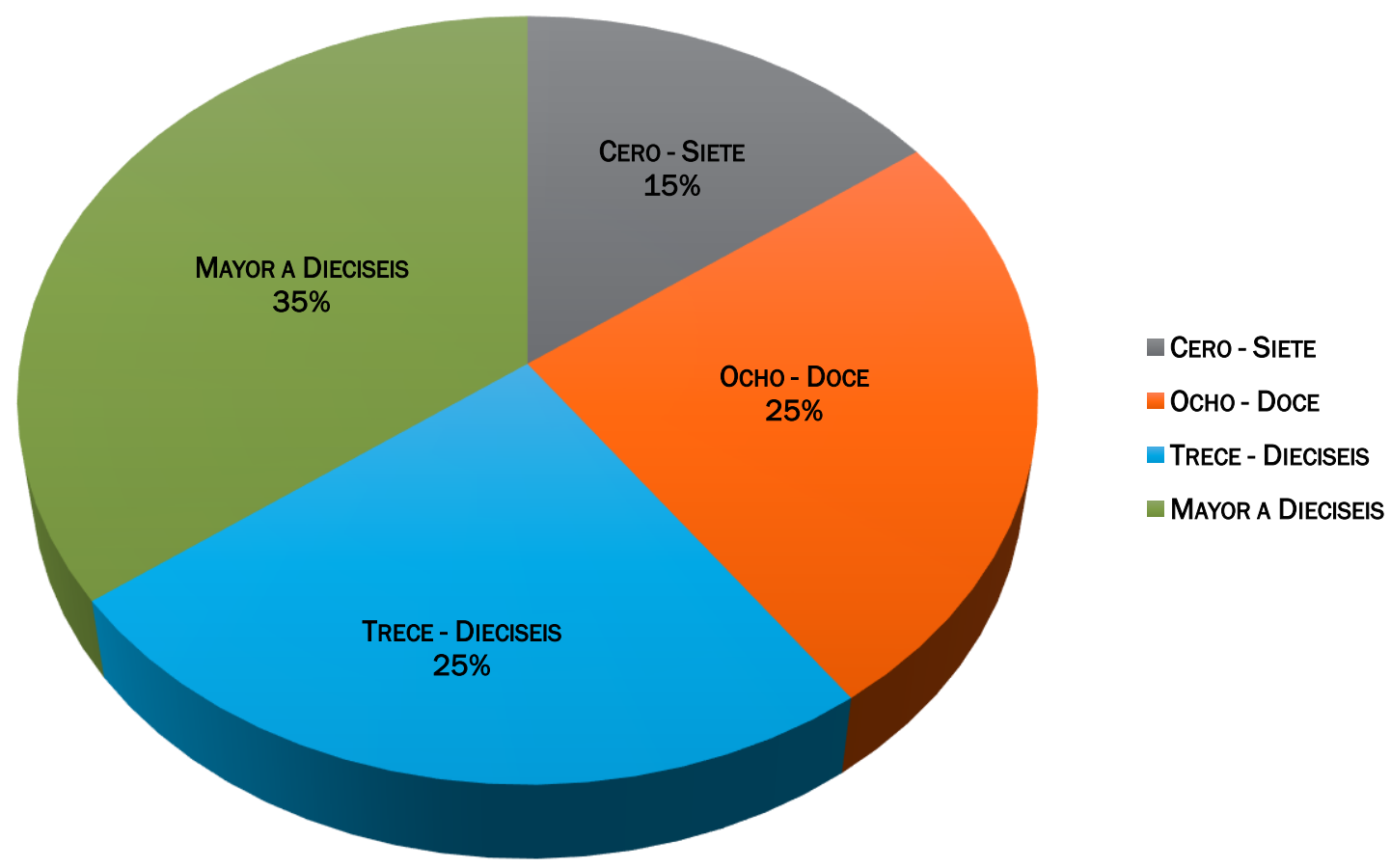

Fuente: Hospital Materno Infantil de Guayaquil - Área de neonatología

Elaborado por: Autores

Análisis: El siguiente gráfico representa las condiciones de salud del neonato con Síndrome de Abstinencia luego de ser valorado con la escala de Finnegan, los datos obtenidos demuestran que el 35\% correspondiente a 7 neonatos tienen una puntuación $>16$ lo que significa una SA severo, seguido de 5 neonatos que representan el 25\% tienen una puntuación entre 13 y 16 lo que significa un SA moderado; 5 neonatos que representan el 25\% tienen una puntuación entre 8 y 12 lo que significa un SA leve y 3 neonatos que representan el 15\% del total poseen una puntuación de 0 y 7 lo que significa que no posee síndrome de abstinencia. 


\section{Cuidados de enfermería en la atención de neonatos con síndrome de abstinencia}

Vol. 2, núm. 3., (2018)

Milka María Lozano Domínguez; Germania Marivel Vargas Aguilar; Crelia Mariana Tufiño Gavidia; María Eiralda Mieles Moreira

\section{Gráfico 3 - Frecuencia de monitoreo de constantes vitales}

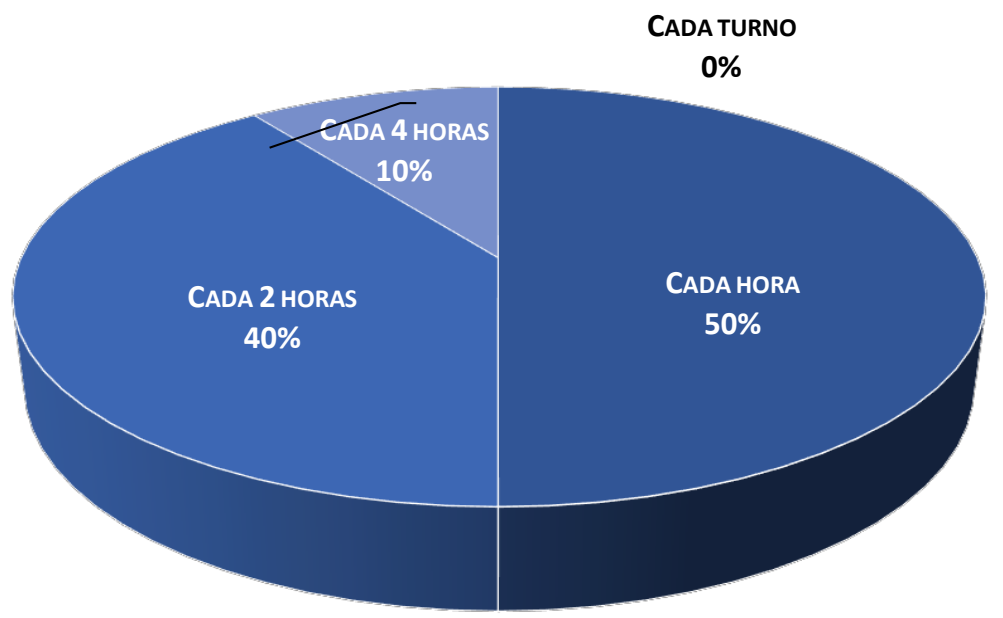

Fuente: Hospital Especializado Mariana de Jesús - Área de neonatología

Elaborado por: Autores

Análisis: El siguiente gráfico representa los datos obtenidos sobre la frecuencia de monitoreo de las constantes vitales, en el cual la población analizada se pudo determinar que efectúa cada hora el monitoreo de los signos vitales correspondiente a un valor porcentual del 50\%, el otro $40 \%$ lo realiza cada dos horas, mientras que un 10\% de la población manifiesta realizar el monitoreo de estas constantes cada 4 horas. 


\section{Cuidados de enfermería en la atención de neonatos con síndrome de abstinencia}

Vol. 2, núm. 3., (2018)

Milka María Lozano Domínguez; Germania Marivel Vargas Aguilar; Crelia Mariana Tufiño Gavidia; María Eiralda Mieles Moreira

\section{Gráfico 4 - Tiempo de consumo de drogas antes del parto}

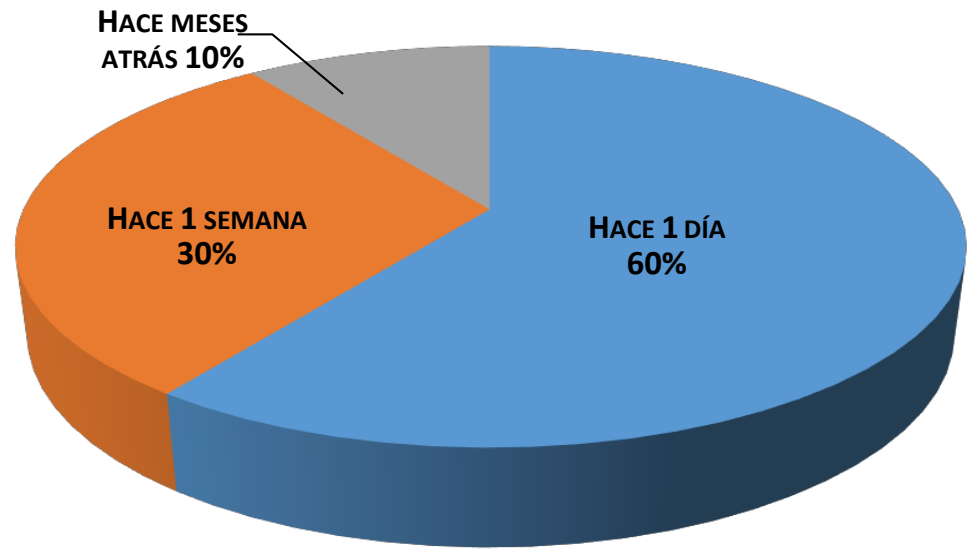

Fuente: Hospital Especializado Mariana de Jesús - Área de neonatología

Elaborado por: Autores

Análisis: Como se puede apreciar en el gráfico del tiempo de consumo de drogas antes del parto, el $60 \%$ de la población estudiada indico que fue hace un día que consumieron heroína, seguido de un $30 \%$ que indico que fue hace una semana, mientras que el 10\% restante de la población manifestó realizar el consumo de heroína hace meses atrás.

Gráfico 5 - Condiciones de salud del recién nacido (según test de APGAR) 


\section{Cuidados de enfermería en la atención de neonatos con síndrome de abstinencia}

Vol. 2, núm. 3., (2018)

Milka María Lozano Domínguez; Germania Marivel Vargas Aguilar; Crelia Mariana Tufiño Gavidia; María Eiralda Mieles Moreira

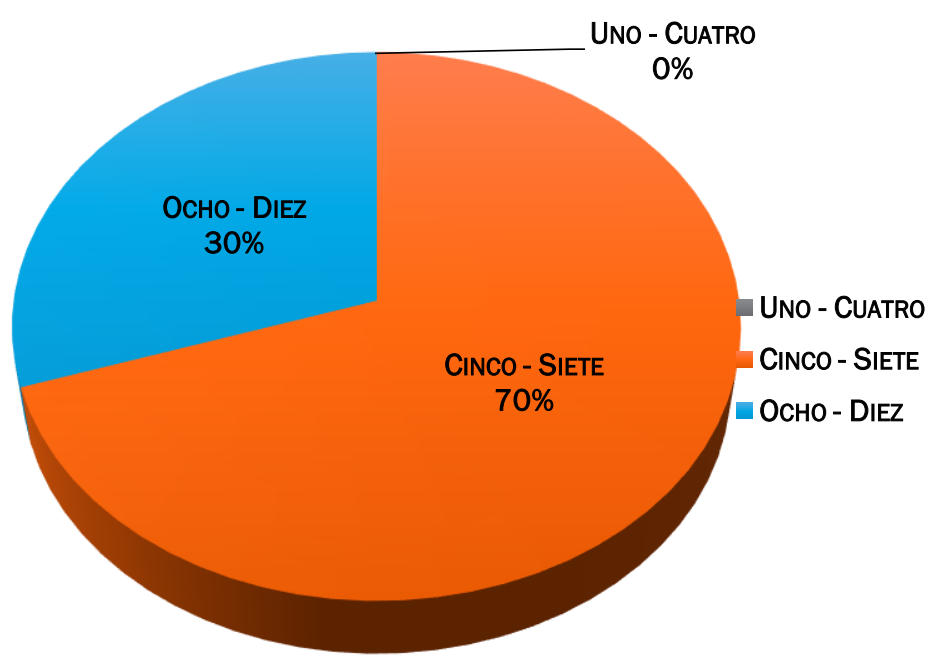

Fuente: Hospital Materno Infantil de Guayaquil - Área de neonatología

Elaborado por: Autores

Análisis: Mediante el gráfico se observa la condición del recién nacido según el Test de Apgar, se determinó que de 14 neonatos que corresponde al 70\% estos se ubican de 5-7 en la escala lo que significa que el RN necesita atención medica mientras que el otro 30\% correspondiente a 6 neonatos, se sitúan en una escala del 8 - 10 que significa un valor normal. 


\section{Cuidados de enfermería en la atención de neonatos con síndrome de abstinencia}

Vol. 2, núm. 3., (2018)

Milka María Lozano Domínguez; Germania Marivel Vargas Aguilar; Crelia Mariana Tufiño Gavidia; María Eiralda Mieles Moreira

\section{Gráfico 6 - Estancia del recién nacido en el área de observación}

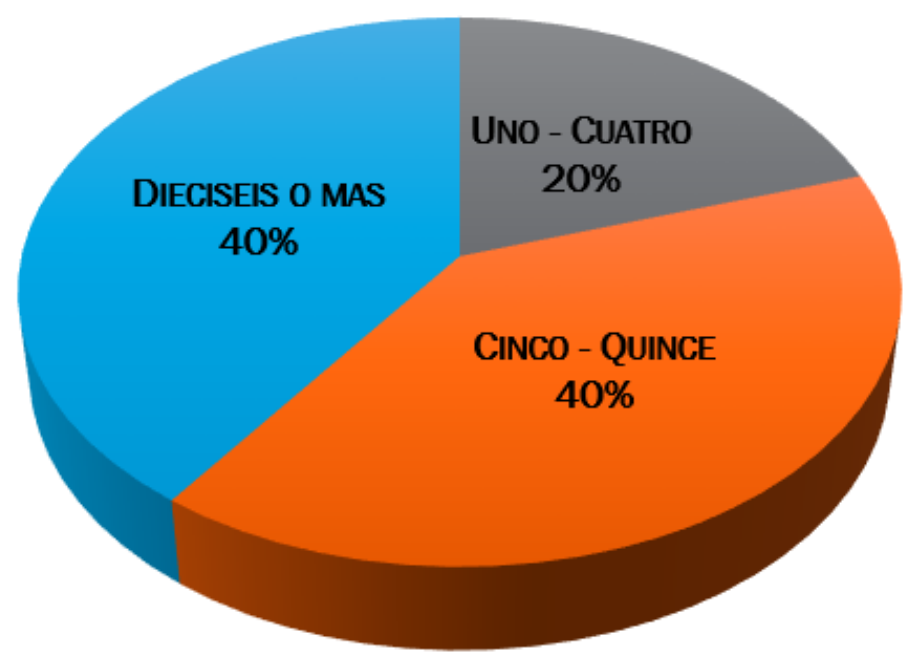

- UnO - CuATRO

CINCO - QUINCE

DIECISEIS O MAS

Fuente: Hospital Materno Infantil de Guayaquil - Área de neonatología

Elaborado por: Autores

Análisis: Según el grafico estadístico de la estancia del neonato en el área de observación, demuestra que el $40 \%$, es decir 8 neonatos permanecen en el área de observación de dieciséis o más días a diferencia del otro $40 \%$ (8) que continúan de cinco a quince días, mientras que el restante de la población corresponde al 20\% (4) los cuales se queden de uno a cuatro días. 


\section{Cuidados de enfermería en la atención de neonatos con síndrome de abstinencia}

Vol. 2, núm. 3., (2018)

Milka María Lozano Domínguez; Germania Marivel Vargas Aguilar; Crelia Mariana Tufiño Gavidia; María Eiralda Mieles Moreira

\section{Gráfico 7 - Bajo peso en el neonato después del parto}

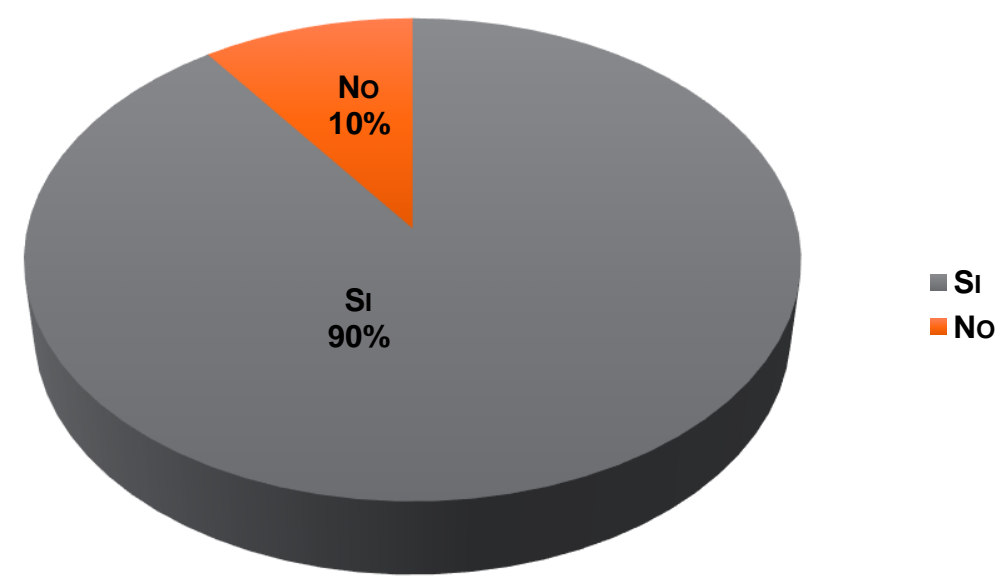

Fuente: Hospital Materno Infantil de Guayaquil - Área de neonatología

Elaborado por: Autores

Análisis: La grafica representa los datos obtenidos sobre el peso del neonato después del parto, en los cuales se pudo determinar en un $90 \%$ que el mayor porcentaje de los recién nacidos de madres consumidoras de heroína si presentan bajo peso, mientras que el otro $10 \%$ de la población en cuestión no presento déficit de peso al nacer. 


\section{Cuidados de enfermería en la atención de neonatos con síndrome de abstinencia}

Vol. 2, núm. 3., (2018)

Milka María Lozano Domínguez; Germania Marivel Vargas Aguilar; Crelia Mariana Tufiño Gavidia; María Eiralda Mieles Moreira

\section{Gráfico 8-Intervenciones de enfermería según el estado de salud del neonato}

Fuente: Hospital Materno Infantil de Guayaquil - Área de neonatología

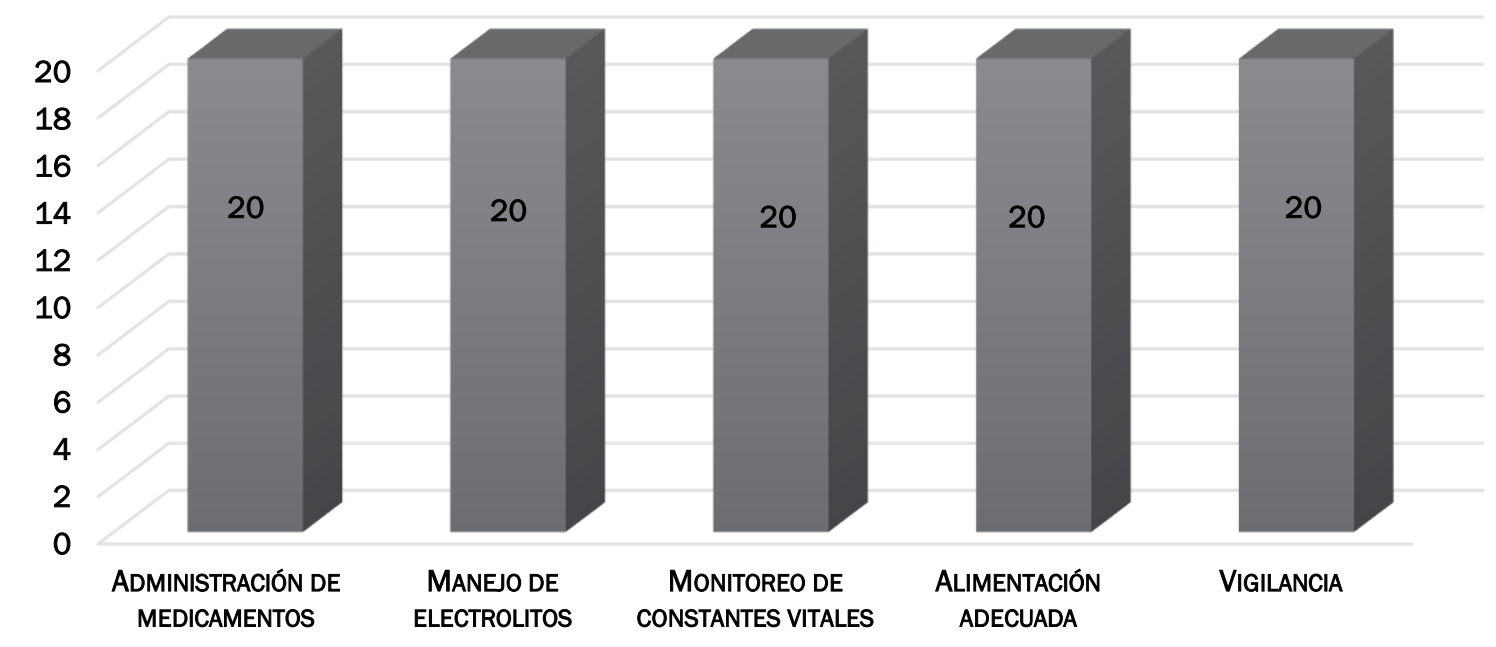

Elaborado por: Autores

Análisis: Observando el gráfico de columnas de los datos obtenidos de las intervenciones de enfermería, se evidencias que dichas actividades como la administración de medicamentos, manejo de electrolitos, monitoreo de constantes vitales, alimentación adecuada y vigilancia, se las desarrollo al $100 \%$ dependiendo de la necesidad del neonato.

\section{Conclusiones.}

Al finalizar con este trabajo de investigación realizado en el Hospital Materno Infantil de Guayaquil, en el área de Neonatología; se contó con tres sujetos de estudio, el personal de enfermería y el neonato crítico y la cuidadora.

Al realizar este estudio se pudo identificar que tanto el niño como la madre con síndrome de abstinencia necesitan tratamiento especializado, por parte del equipo de salud. El niño al presentar 


\section{Cuidados de enfermería en la atención de neonatos con síndrome de abstinencia}

Vol. 2, núm. 3., (2018)

Milka María Lozano Domínguez; Germania Marivel Vargas Aguilar; Crelia Mariana Tufiño

Gavidia; María Eiralda Mieles Moreira

una condición fisiológica delicada requiere cuidados de calidad, mientras que las madres necesitan asesoría psicológica debido a la inconformidad y resistencia que presentan al tratar estos temas. Sin embargo, conforme se desarrolló la investigación, se determinó el rol de enfermería en este tipo de patologías ,en lo que concierne a los diagnósticos de enfermería prevalentes en este síndrome son patrón de alimentación ineficaz, riesgo desequilibrio electrolítico, deterioro del patrón sueño; la mayoría de neonatos en estudio según la escala de Finnegan se encuentran en el indicador > de 16 presentando un síndrome de abstinencia severa, en cuanto a la frecuencia de toma de signos vitales de preferencia se lo realiza cada hora; en tanto a la frecuencia del consumo de drogas antes del parto el gran porcentaje de madre consumieron la sustancia un día antes de su parto. En las condiciones del neonato, el estado de salud, la estancia del RN en el área de neonatología, se puede deducir según los datos obtenidos en el estudio que la mayoría necesitan atención medica especialidad, por lo que requiere una estancia de hospitalaria > a 5 días, presentando bajo peso al nacer, debido a estos resultados los cuidados requeridos, en estos usuarios deben ser cumplidos en un $100 \%$ manteniendo estándares de calidad.

\section{Recomendaciones.}

Se recomienda estandarizar cuidados e elaborar protocolos de atención de enfermería basada en la taxonomía de forma estandarizada que promueva la administración de cuidados fundados en conocimientos científicos que permita satisfacer de manera integral para atender este neonato con calidad y calidez.

Establecer un programa educativo con énfasis en la promoción y fomento de la salud con el apoyo del departamento de psicología, motivando a la participación en esta población. También 


\section{Cuidados de enfermería en la atención de neonatos con síndrome de abstinencia}

Vol. 2, núm. 3., (2018)

Milka María Lozano Domínguez; Germania Marivel Vargas Aguilar; Crelia Mariana Tufiño Gavidia; María Eiralda Mieles Moreira

otras de las recomendaciones, es permitir que el Hospital Materno Infantil de Guayaquil capacite a las madres a través de talleres de inducción acerca de los peligros y complicaciones que tiene el producto en el proceso de embarazo y después del parto por el consumo de estupefacientes.

\section{Bibliografía.}

1. Martín Mardomingo MA, Solís Sánchez G, Málaga Guerrero S, Cuadrillero Quesada C, Pérez Méndez C, Matesanz Pérez JL. Consumo de drogas durante el embarazo y morbilidad neonatal: cambios epidemiológicos en los últimos 10 años. AnPediatr 2003; 58(6):574-579.

2. Cuesta Miguel MJ, Espinosa Briones AB, Val Saurí C. Síndrome de Abstinencia Neonatal. Enfermería Integral 2013 Diciembre 2013(103):24-28

3. Rite Gracia S, Fernández Lorenzo JR, Echaniz Urcelay I, BotetMussons F, Herranz Carrillo G, Moreno Hernando J, et al. Health care levels and minimum recommendations for neonatal care. An Pediatr (Barc) 2013 Jul; 79(1):51.e1-51.e11.

4. World Health Organization [sede web]. HealthTopics. 2014 [acceso 20 de enero de 2014]. Disponible en: http://www.who.int/topics/infant_newborn/en/

5. Mellado JE, Pastor Rodríguez JD, Del Cerro Ortuño F, De ArdanazJorreto S, López Ibáñez, M. Manejo y control del síndrome de abstinencia. Enfermería Global: Revista electrónica semestral de enfermería 2008;7(12).

6. Backes CH, Backes CR, Gardner D, Nankervis CA, Giannone PJ, Cordero L. Neonatal abstinence syndrome: transitioning methadone-treated infants from an inpatient to an outpatient setting. J Perinatol 2012 Jun;32(6):425-430.

7. Nelson MM. Neonatal Abstinence Syndrome: The Nurse's Role. INT J CHILDBIRTH EDUC 2013;28(1):38-42.

8. Hudak ML, Tan RC, The Committee on Drugs, The Committee on Fetus and Newborn. Neonatal Drug Withdrawal. Pediatrics 2012 February 01;129(2):e540-e560.

9. José Alfonso Gutiérrez-Padilla, Andrés A. González-Garrido, Fabiola R. GómezVelázquez, Martha de la Torre-Gutiérrez, Luis Manuel Ávalos Huizar, Héctor García 


\section{Cuidados de enfermería en la atención de neonatos con síndrome de abstinencia}

Vol. 2, núm. 3., (2018)

Milka María Lozano Domínguez; Germania Marivel Vargas Aguilar; Crelia Mariana Tufiño Gavidia; María Eiralda Mieles Moreira

Hernández, David Rodríguez Medina. Hijos de madres adictas con síndrome de abstinencia en Terapia Intensiva Neonatal. Mex. vol.65 no.4 México jul./ago. 2008.

10. José Alfonso Gutiérrez-Padilla, Horacio Padilla-Muñoz, Hugo Gutiérrez-González, Juan Carlos Barrera-de León, Óscar Miguel Aguirre- Jáuregui, Ricardo Martínez-Verónica, Iván Gutiérrez-González, Luis Manuel Ávalos-Huizar. Evolución clínica de hijos de madres con adicciones internados en una unidad de terapia intensiva neonatal del Occidente de México. Medigraphic. Ginecol Obstet Mex 2014; 82 (07) 